\title{
Mass Spectra of 3-Methylcholanthrene and Some Related Compounds
}

\author{
Gonya TAKahashi* AND AKIRA Kato**
}

(Received 19 July 1972)

\begin{abstract}
Data are given of the mass spectra of 3-methylcholanthrene, one of the strongest carcinogenic hydrocarbons, and some related compounds. The structures of their fragments are shown speculatively.
\end{abstract}

\section{Introduction}

Many studies on the metabolism of polyaromatic hydrocarbons have been reported from interest in carcinogenicity. In the previous investigation ${ }^{1)}$ on the metabolism of 3-methylcholanthrene (3-MC), one of the strongest carcinogenic hydrocarbons, we detected some metabolites of this chemical substance in the feces of mice. These compounds have been detected with conventional methods. Recently, a combining system of gas chromatography and mass spectrometry has been used widely in other studies but rarely in polyaromatic hydrocarbons. As a preliminary investigation of the detection of these compounds using this system, we have studied the mass spectrometry of 3-MC and some related compounds.

This paper gives mass spectral data of 3-MC and related compounds, and speculative structures of their fragments.

\section{Results and Discussion}

The mass spectra and speculative structures of the fragments are shown in Figs. 1 to 11 . When the metastable ions in fragmentation were observed, they were shown as $m^{*}$ in these figures.

\subsection{3-MC (Figs. 1 and 2)}

The mass spectrum is very simple with a few fragment ions and the strong molecular ion which is the base peak. The peak at $m / e 267$ may be caused by the tropilium ion formed by ring expansion as shown by the fact that an analogous peak was found at $m / e(M-1)$ for methyl acenaphthylenes. ${ }^{2)}$ In the higher mass range, one of the main peaks at $m / e 253$ ( $\mathrm{m}^{*} 238.7$, calc. 238.8 ) is due to an ion formed with loss of the methyl radical from the parent ion, and the other at $\mathrm{m} / \mathrm{e}$ 252 ( $\mathrm{m}^{*} 250.8$, calc. 251.0) does from loss of the hydrogen radical. In the intermediate mass range all peaks are small, and in the lower mass range there are prominent series of doubly charged ion peaks.

\subsection{1-Hydroxy-3-MC (Figs. 3 and 4)}

The peak at $m / e 284$ is due to the molecular ion, the relative abundance of which increases when the energy of the electron beam is reduced to $12 \mathrm{eV}$. The base peak at $m / e ~ 266$ ( $\mathrm{m}^{*} 249.1$, calc. $249.1)$ is due to mainly to an ion formed

* Chest Disease Research Institute, Kyoto University (Sakyo-ku, Kyoto, Japan).

** Faculty of Pharmaceutical Sciences, Kyoto University (Sakyo-ku, Kyoto, Japan). 


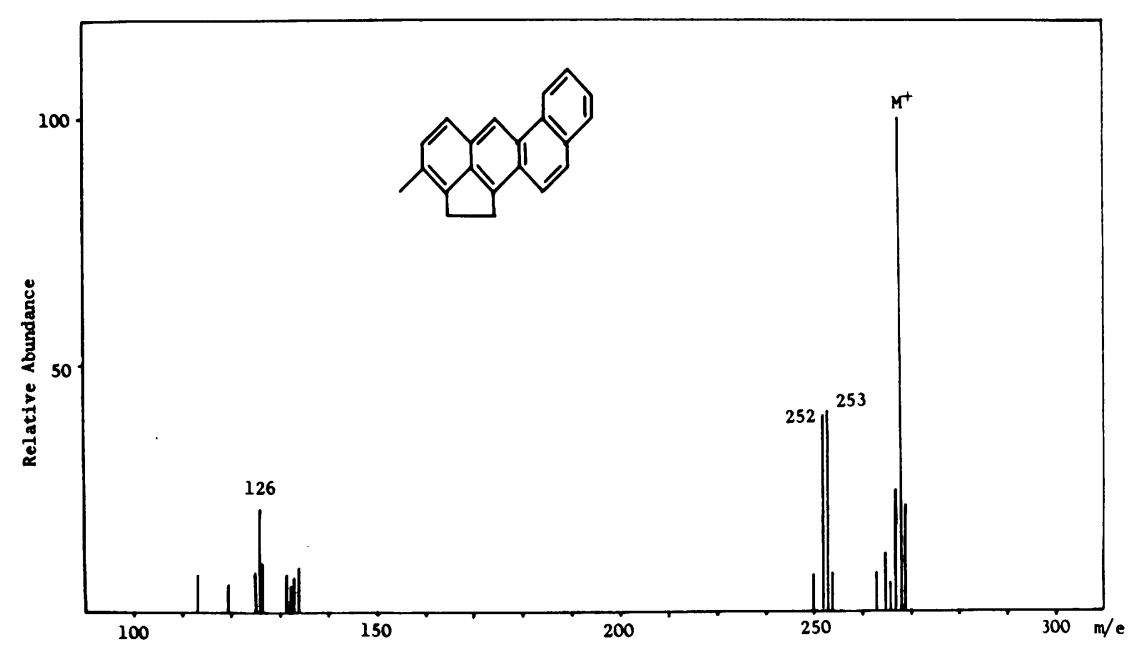

Fig. 1. Mass spectrum of 3-methylcholanthrene.

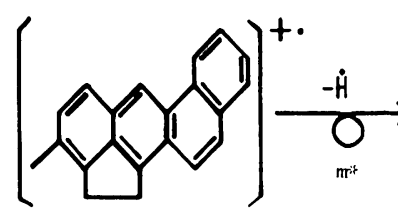

$\mathrm{m} / \mathrm{c} 268$

$\downarrow-\dot{\mathrm{C}} \mathrm{H}_{3}$

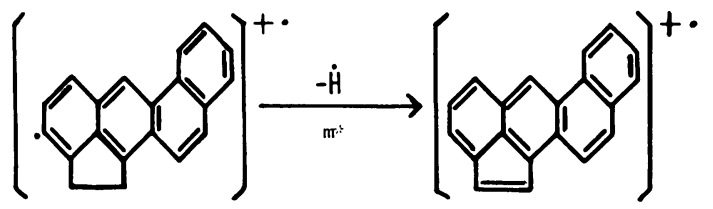

$m /: 253$

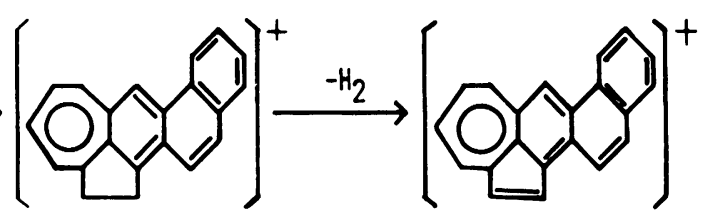

$\mathrm{m} /=267$

$n /=265$

$\mathrm{m} / \mathrm{s} 252$

Fig. 2. Speculative structures of fragments in 3-methylcholanthrene.

by dehydration from the parent ion. Ions containing tropilidene and dehydrotropilidene structures are apparent to recognize. The peaks at $m / e 241$ and 239 are probably due, respectively, to ions formed with loss of acetylene and ethylene from the cyclopentene ring of the cholanthrene structure. In the lower mass range, doubly charged ion peaks appear prominently.

\subsection{2-Hydroxy-3-MC (Fig. 5)}

The mass spectra of 1 - and 2-hydroxy3-MC show almost the same fragmentation pattern, except for some differences between relative abundance of peaks. The base peak is the molecular ion peak at $m / e$ 284. The speculative fragmentation is the same as that of 1-hydroxy-3-MC.

\subsection{1-Keto-3-MC (Figs. 6 and 7)}

In the higher mass range, the molecular 
Mass Spectra of 3-Methylcholanthrene and Some Related Compounds

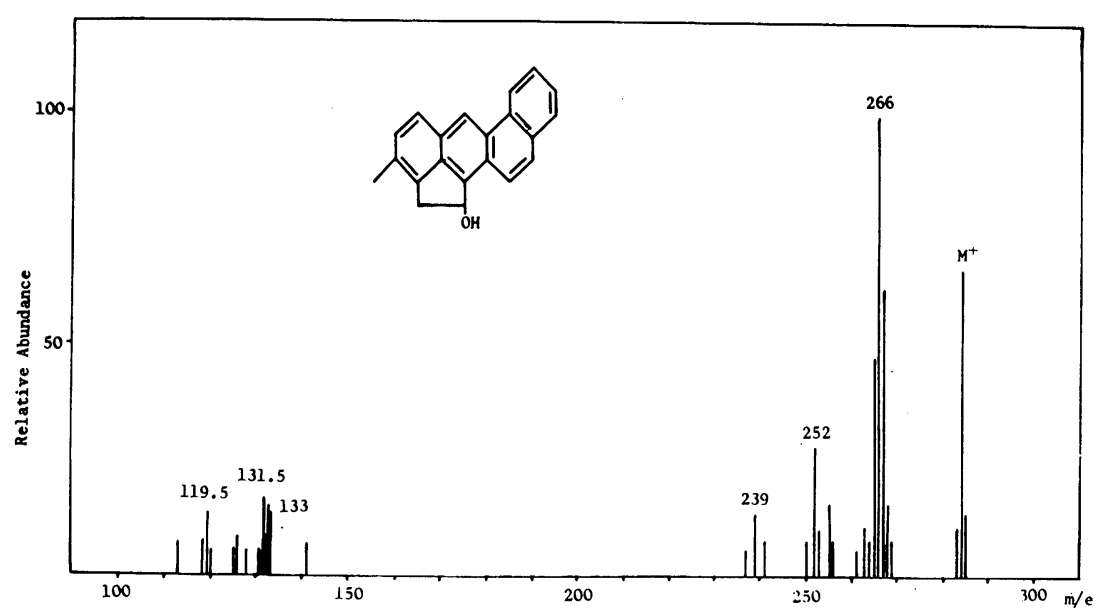

Fig. 3. Mass spectrum of 1-hydroxy-3-methylcholanthrene.
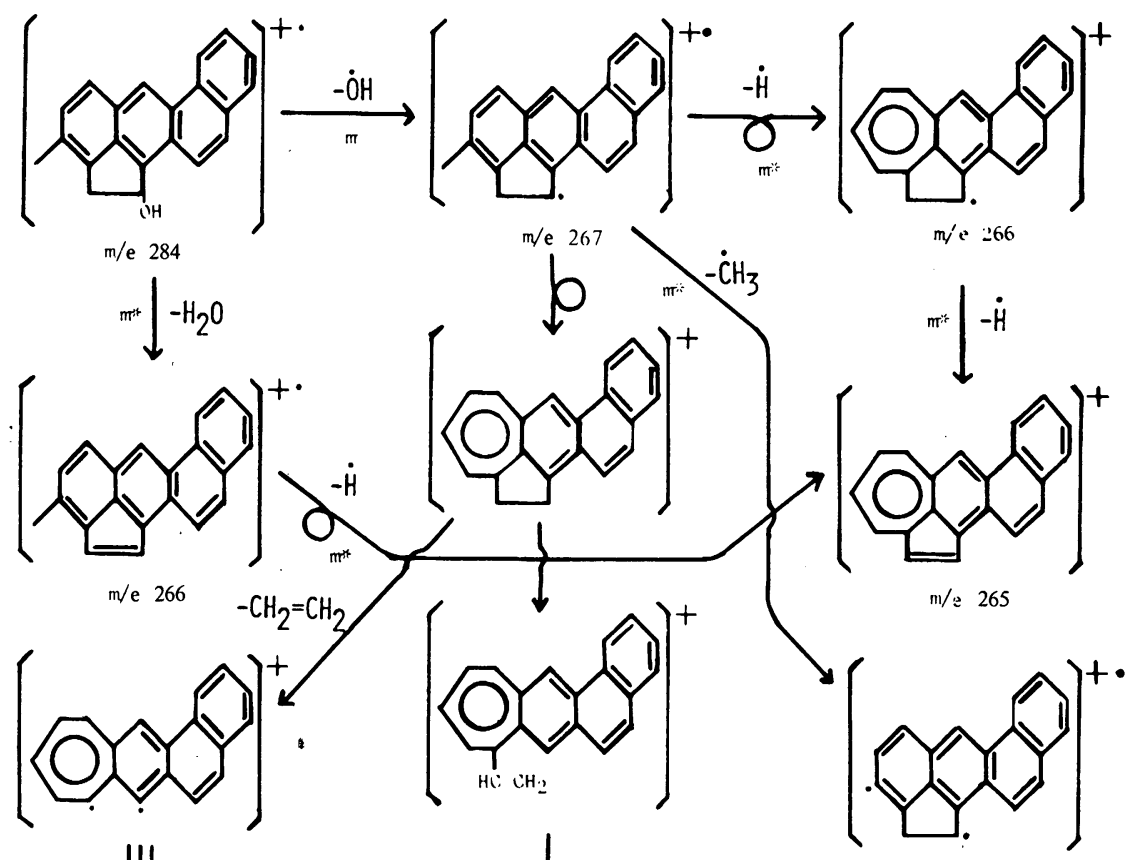

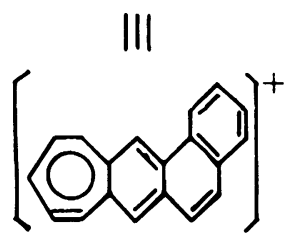

$\mathrm{m} / \mathrm{e} 239$

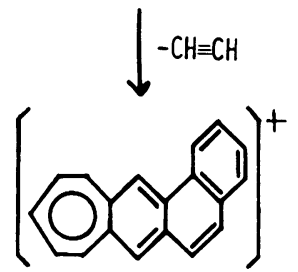

m/e 241
III

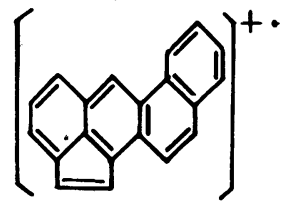

$\mathrm{m} / \mathrm{e} \quad 252$

Fig. 4. Speculative structures of fragments in 1-hydroxy-3-methylcholanthrene. 


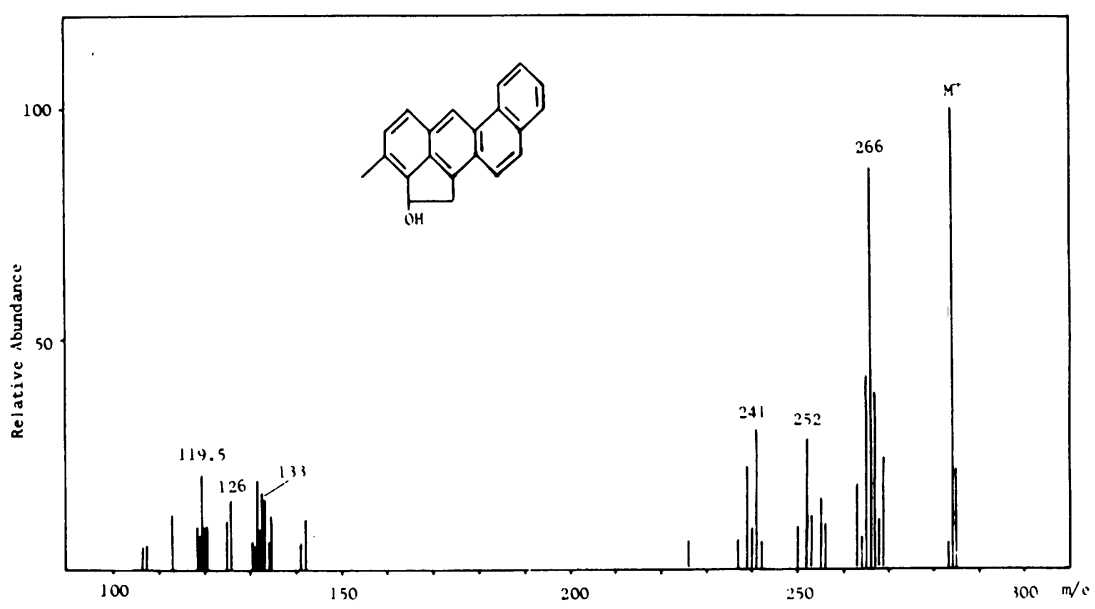

Fig. 5. Mass spectrum of 2-hydroxy-3-methylcholanthrene.

ion gives the strong peak at $m / e 282$. The peak at $m / e 254$ may be due to an ion containing tropilidene. The peaks at $m / e 252$ and 239 are probably due to ions containing dehydrocyclooctatetraenei and dehydrotropilidene, respectively. The peak at m/e 141 must be due to a doubly charged ion of the parent because there is a peak at $m / e 141.5$, which is due to a doubly charged ion of the $(M+1)$, and the ratio of the heights of $m / e 141$ to 141.5 is the same as the ratio of the parent ion to the $(M+1)$ ion.

\subsection{1, 2-Dihydroxy-3-MC (Figs. 8 and 9)}

The base peak is the molecular ion peak at $m / e 300$. The peak at $m / e 282$ ( $m^{*} 264.8$, calc. 265.1 ) is due to an ion formed by dehydration from the parent ion. The peak at $m / e 269\left(\mathrm{~m}^{*} 241.0\right.$, calc. 241.2 ) is due to an ion formed with loss of the methoxy radical which was caused by rearrangement of the methyl radical into the 2-hydroxy radical. The tropilium and its benzyen type ions also appear at $m / e ~ 253,239,228$. The peaks at $m / e$ 228 (D, m/e 229) and 226 (without containing deuterium) are probably due, respectively, to ions formed with loss of acetylene from one of the aromatic nuclei and cyclopentene.

\subsection{1, 12-Dihydro-11, 12-dihydroxy- 3-MC(Figs. 10 and 11)}

As shown in Fig. 11, the three cases are possible in rearrangement of the deuterium-labeled ions. The peak at $m / e$ 302 is due to the molecular ion. The base peak at $m / e 284\left(m^{*} 267.1\right.$, calc. 267.1) is due to an ion formed by dehydration from the parent ion. The peak at $m / e 269$ ( $\mathrm{m}^{*} 254.7$, calc. 254.8 ) is due to loss of the methyl radical from the fragment at $m / e$ 284. The peak at $m / e 241$ (D, m/e $242 ; \mathrm{m}^{*} 215.6$, calc. 215.9 ) is due to an ion containing fluorene which is formed with loss of $\mathrm{CO}$ from the hydroxy group of the fragment at $m / e 269(\mathrm{D}, m / e$ 270). It is impossible to recognize an ion containing biphenylene arising from fluorene as shown in Fig. 12. The peak at m/e 242 is expected to be an ion of biphenylene derivative which is formed with loss of ethylene diol, and it decomposes finally to the tropilium ion and its benzyen type ion, taking their places at $m / e 239$ and 226. 


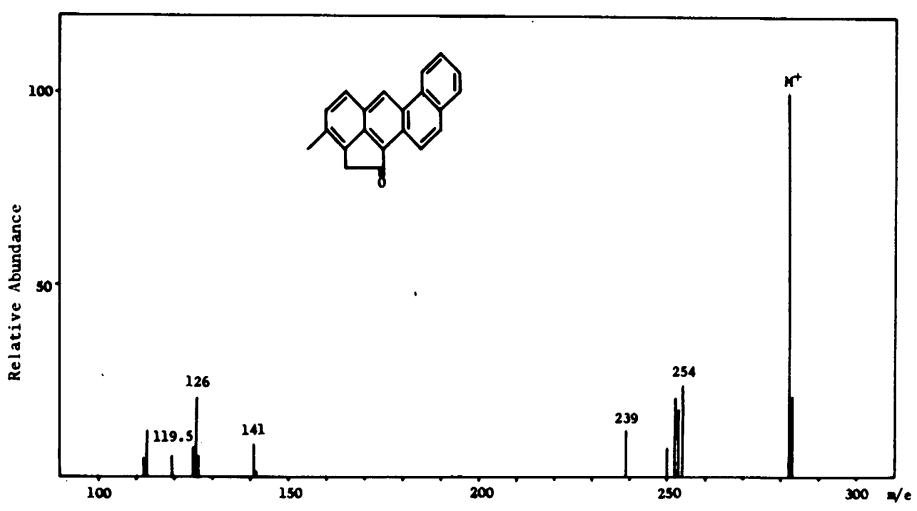

Fig. 6. Mass spectrum of 1-keto-3-methylcholanthrene.

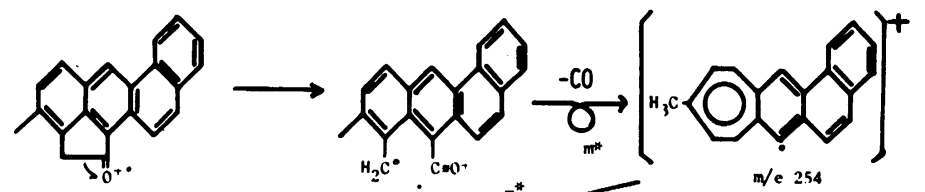

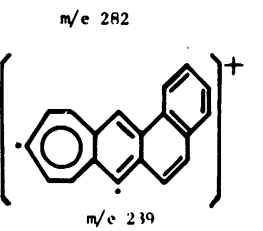

III

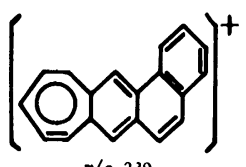

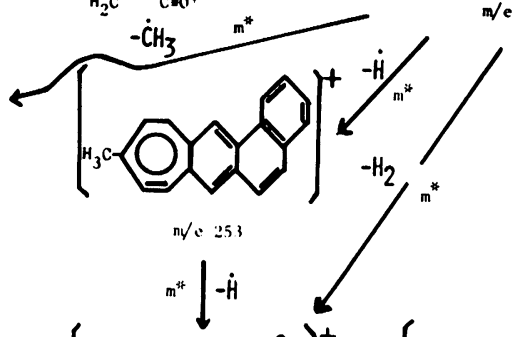

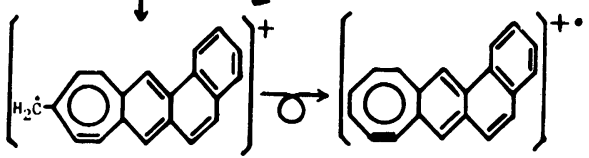

$m / 252$ $m / e 252$

Fig. 7. Speculative structures of fragments in 1-keto-3-methylcholanthrene.

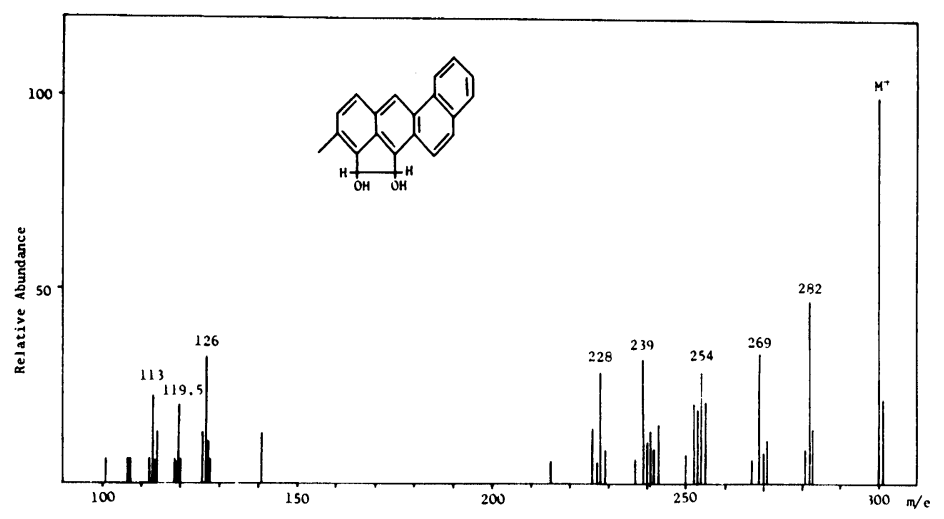

Fig. 8. Mass spectrum of 1, 2-dihydroxy-3-methylcholanthrene. 

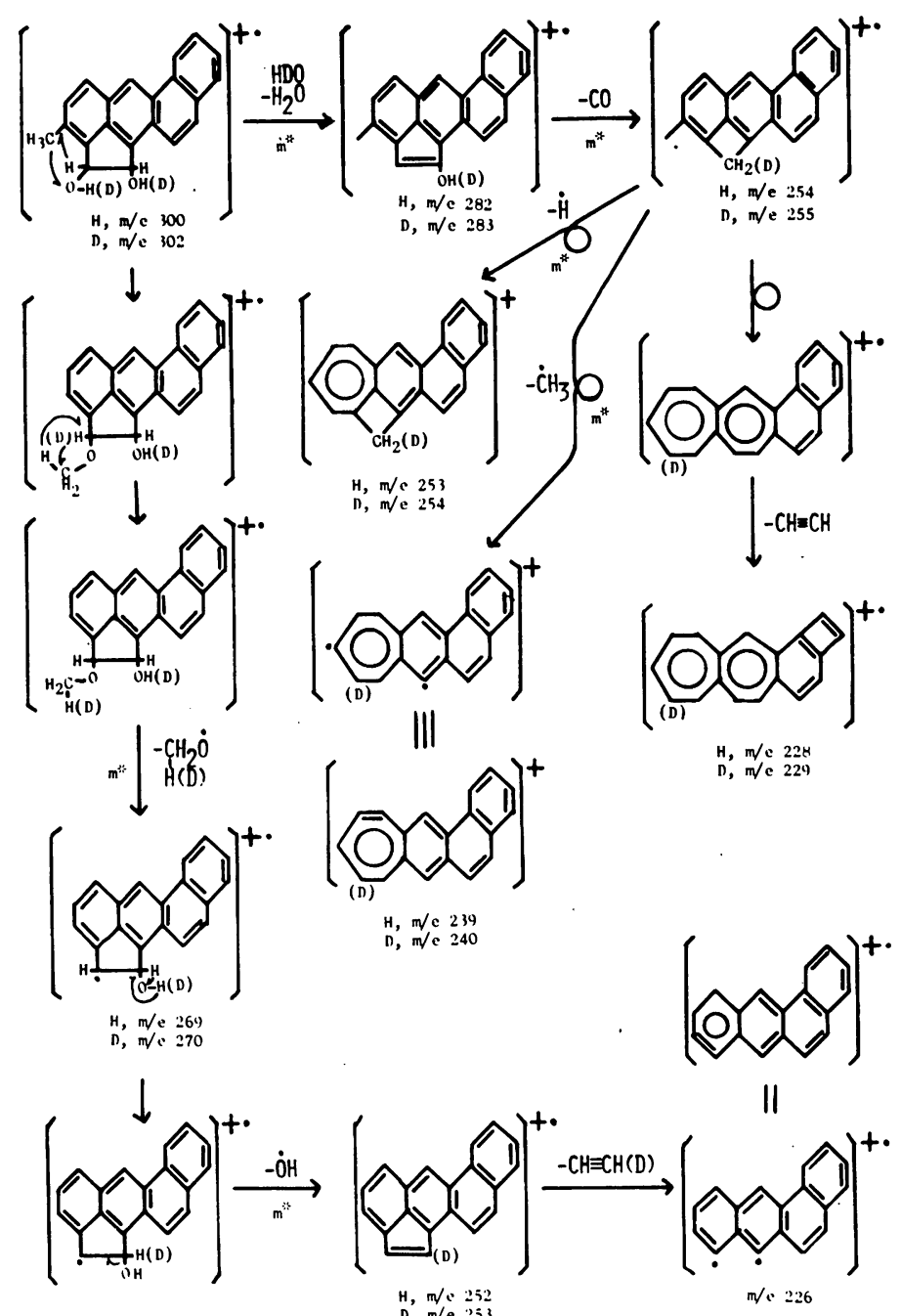

Fig. 9. Speculative structures of fragments in 1, 2-dihydroxy-3-methylcholanthrene.

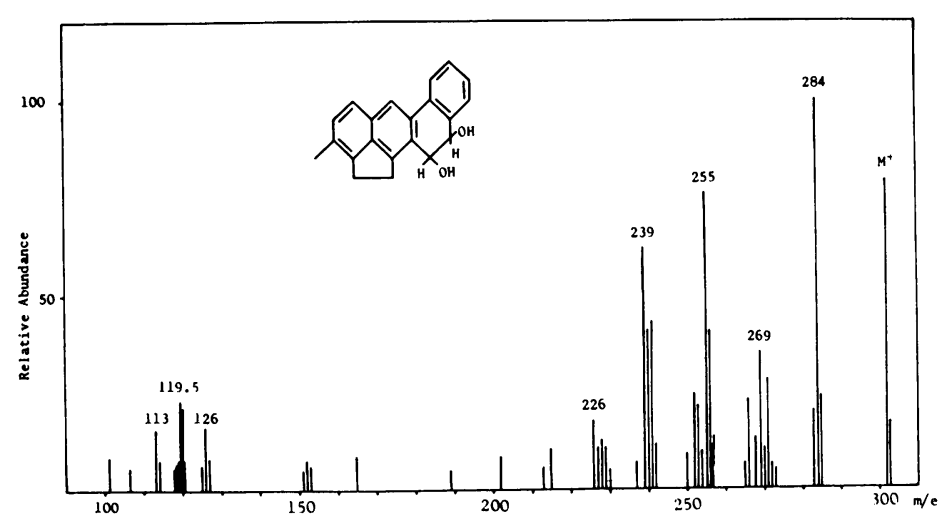

Fig. 10. Mass spectrum of 11,12-dihydro-11, 12-dihydroxy-3-methylcholanthrene. 
A)

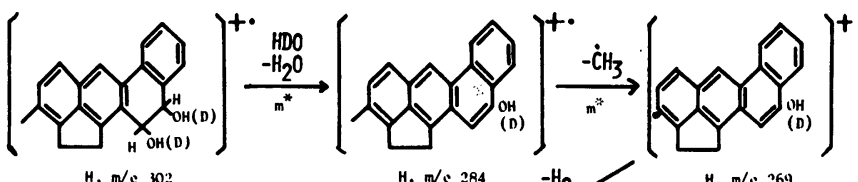

$\begin{array}{ll}H, \mathrm{~m} / \mathrm{e} & 302 \\ \mathrm{D}, \mathrm{m} / \mathrm{C} & 104\end{array}$

$H, m /: 284$
$D, m /: 285$

$H, m /: 269$
$D, m /: 270$
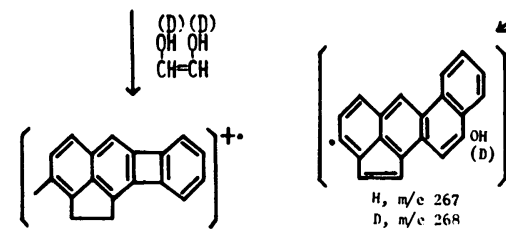

$m^{*} \downarrow-\mathrm{CO}$

H, $m /$ e 267

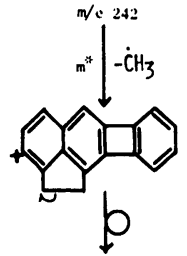

b
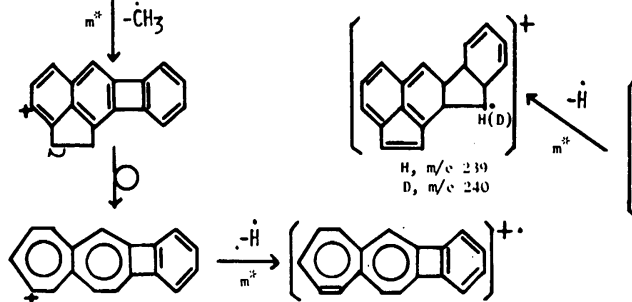

m/: 227

$\stackrel{-\dot{H}}{m^{*}}(O)$

$m / 2: 26$

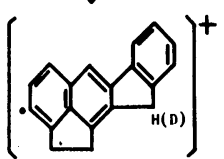

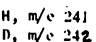

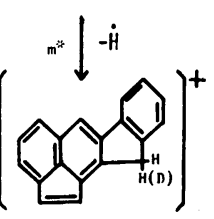

$H, m /=240$

B)

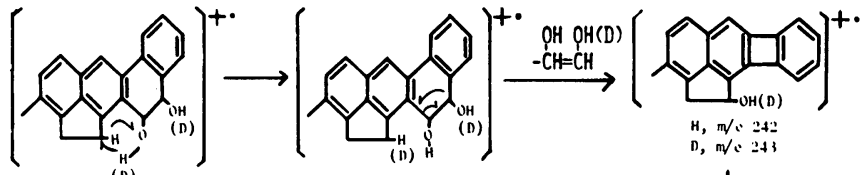

$\begin{array}{lll} & \text { (D) } \\ H, & m /: & 30: \\ \text { D), } & m / 2 & 304\end{array}$

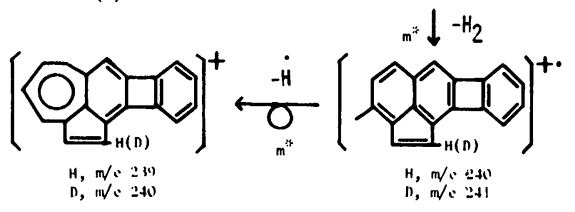

c)

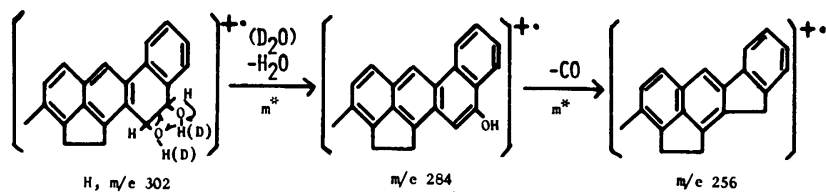

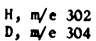

nae 256

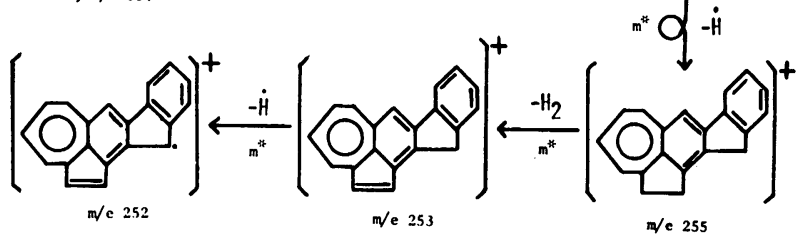

Fig. 11. Speculative structures of fragments in 11, 12-dihydro-11, 12-dihydroxy-3-methylcholanthrene. 


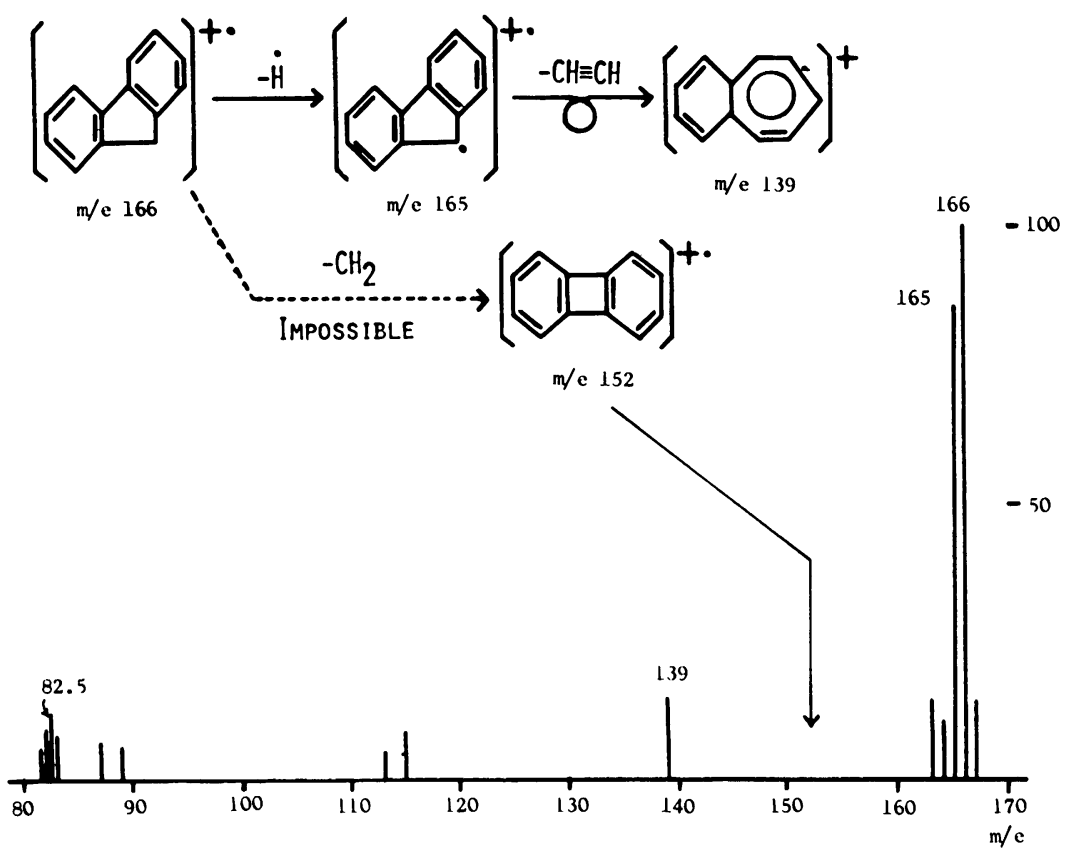

Fig. 12. Mass spectrum and speculative structures of fragments of fluorene.

\section{Experimental}

\subsection{Apparatus}

Mass spectra were measured by a modified Hitachi RMU-6C mass spectrometer equipped with the direct inlet system. Ionizing voltage: $70 \mathrm{eV}$; total emission: $80 \mu \mathrm{A}$; ion source temperature: $200^{\circ} \mathrm{C}$

\subsection{Reagents}

3-MC was obtained from Fluka (CH9470 Buchs, Schwizerland).

1-Keto-3-MC was prepared by dichromate oxidation of $3-\mathrm{MC}$ by the method of Fieser et al. ${ }^{3)}$ The crude product was purified and finally crystallized from ether to yield yellow needles, m. p. $261.7^{\circ} \mathrm{C}$, according to the method of Sims. ${ }^{4)}$

1-Hydroxy-3-MC - 1-Keto-3-MC was reduced with lithium tetrahydroaluminate according to Sims. ${ }^{4)}$ The crude product was purified by chromatography on silica gel in benzene and crystallized from ethanol to yield 1-hydroxy-3-MC white needles, m. p. $210.5^{\circ} \mathrm{C}$ (decomp.).

1, 2-Dihydroxy-3-MC was prepared by acetylation of 3-MC by lead tetraacetate in hot acetic acid and by hydration of the product by potassium hydroxide according to the method of Sims. ${ }^{4)}$ The crude product was chromatographed in benzene-acetone $(9: 1)$ on silica gel. The two violet fluorescent bands were evaporated and crystallized from ethanol to yield cis- and trans-1, 2-dihydroxy-3-MC both in microscopically visible brown needles. The melting point was uncertain because of decomposition into 2-keto-3-MC at $190-200^{\circ} \mathrm{C}$. The substance obtained from the fast moving band was proved to be the cis type from production of the isopropylidene derivative with acetone, and the one obtained from the second band was proved to be the trans type from no 
production of this derivative. In our experiment the trans type was used.

2-Hydroxy-3-MC-According to the method of Sims,") 2-hydroxy-3-MC was obtained by the reduction of 2-keto-3-MC in the same manner as preparation of 1-hydroxy-3-MC from 1-keto-3-MC. The melting point of this product was uncertain because it seemed to change to an another compound at $180^{\circ} \mathrm{C}$ which melted at $219.6^{\circ} \mathrm{C}$ (decomp.).

11, 12-Dihydro-11, 12-dihydroxy-3-MC was prepared by $\mathrm{OsO}_{4}$ oxidation according to the description by Cook and Schoental. ${ }^{5)}$ The crude product was purified by chromatography on silica gel in benzeneacetone $(9: 1)$ and was crystallized from ethanol to yield 11, 12-dihydro-11, 12 . dihydroxy-3-MC in white powder which turned to red at $190^{\circ} \mathrm{C}$ and melted at $202^{\circ} \mathrm{C}$ (decomp.).
The deuterium-labeled compounds were obtained by mixing deuterium oxide and the chemicals.

All compounds prepared were identified with the standards described by Sims) by UV absorption spectra and $R_{\mathrm{f}}$ values of thin layer chromatography on silica gel, and their purities were confirmed by disappearance or.decrease of the peaks except for the molecular ion when the energy of the electron beam is reduced to $12 \mathrm{eV}$.

\section{References}

1) G. Takahashi and K. Yasuhira, Cancer Res., 32, 710 (1972).

2) I. D. Entwistle and R. A. W. Johnstone, J. Chem. Soc., 1968, 1818.

3) L. F. Fieser and E. B. Hershberg, J. Am. Chem. Soc., 60, 2542 (1938).

4) P. Sims, Biochem. J., 98, 215 (1966).

5) J. W. Cook and R. Schoental, J. Chem. Soc., 1948, 170. 